\title{
Expedited organ donation in Victoria, Australia: donor characteristics and donation outcomes
}

Rohit L D'Costa, Samuel Radford, Helen I Opdam, Mark McDonald, Leanne McEvoy and Rinaldo Bellomo www.doi.org/10.51893/2020.4.oa2

Published online first 7 December 2020

Despite significant recent increases in the number of deceased organ donors in Victoria, Australia, ${ }^{1}$ there remains an unmet demand, with many patients on the transplantation waiting list. ${ }^{2}$ Among a number of measures introduced in 2015 aimed at increasing the potential donor pool was the introduction of a guideline for the donation specialist nursing coordinators (DSNCs), who undertake the process of donation work-up, to more rapidly coordinate retrieval surgery in selected instances. The two main scenarios envisaged for this guideline were for donors determined by the intensivist to be at high risk of cardiac arrest or failed physiological support within the usual timeframes for workup, or where the family or the legally responsible person ${ }^{3}$ were not able or willing to wait the usual time frame for retrieval to occur (and would otherwise not consent to donation). Typically, between the time of obtaining formal consent and commencement of the retrieval procedure, the complex process of obtaining medical and social history data, reviewing the medical records, organising blood and imaging tests required for determining organ suitability, and entering relevant data into the electronic donor record takes around 24 hours. The expedited guideline aimed to reduce this time to less than 6 hours.

The guideline aimed to limit the steps in the workup process to those essential for establishing a high likelihood of donation, with organ referral and allocation deferred to the period after retrieval surgery. In contrast, the usual pathway is for referrals with complete donor information to recipient units - made on the basis of nationally agreed rules of priority 4 - and acceptance of these offers before retrieval is scheduled. It was anticipated that the expedited guideline would be particularly suited to kidney donation, as tolerable cold ischaemia times for kidneys are much longer and allow enough time after retrieval for collation and sharing of donor information, as well as management of transport and recipient logistic factors. However, consideration of non-renal organ or tissue referral was not excluded.

\begin{abstract}
Background: Deceased organ donation work-up typically takes 24 hours or more. Clinicians may thus discount the possibility of donation when the potential donor is physiologically unstable or family requirements do not allow this length of time. This may lead to loss of transplantable organs. In 2015, we introduced an expedited work-up guideline with the aim of facilitating donation in these circumstances and maximising donation potential.

Objective: To determine the number of expedited work-up (consent to retrieval procedure of 6 hours or less) donors from 2015 to 2018, compare their clinical and demographic characteristics with standard donors, and assess the outcome of transplanted organs and organ recipients.

Design: We performed a retrospective audit of the electronic database for all Victorian donors from 2015 to 2018. We obtained transplant outcome data from the Australia and New Zealand Dialysis and Transplant Registry (ANZDATA).

Results: Overall, 38 expedited pathway donors donated 78 organs for transplantation (70 kidneys, four lungs, three livers, one pancreas). Of these, 55 retrieved kidneys were successfully transplanted. The lungs, livers and pancreas retrieved were all transplanted. For the kidney recipients, early graft dysfunction requiring dialysis was more common than with organs from the standard pathway $(71 \% v$ 38\%; $P<0.0001)$; however, short and medium term graft and patient survival were similar. Three recipients from the expedited pool experienced graft failure and two subsequently died. Of the two lung recipients, one died at day 622 of chronic rejection.

Conclusions: Expedited pathway donation is feasible with acceptable donation outcomes. Clinicians should consider donation even when physiological instability or family requirements preclude standard organ donation work-up times.
\end{abstract}




\section{ORIGINAL ARTICLES}

During the work-up period, donor physiological management is directed by intensive care specialists according to national guidelines, ${ }^{5}$ with low rates of failed physiological support (<1\% of consented donors). ${ }^{6}$ By being able to rapidly work up a potential donor, we hoped that concern about inability to physiologically support a patient for 24 hours, or family requirements that precluded the availability of this length of time before the organ donation procedure would not result in a decision to exclude such patients for consideration of donation.

We hypothesised that expedited work-up of donors would yield meaningful donation outcomes both in terms of organs transplanted as well as graft and patient survival. Accordingly, we examined patient clinical characteristics and kidney (and other organ) donation outcomes over a 4-year period for cases where organ donation occurred within the above expedited time frame and compared such outcomes to the broader donor pool.

\section{Expedited donor guideline}

Key clinical and logistic elements of the expedited pathway and differences from the standard coordination pathway are highlighted in Table 1. The main focus of the expedited guideline is to prioritise tasks vital to donation, including rapid donor suitability assessment, obtaining legal consents, and scheduling organ retrieval surgery. Gathering of more detailed donor information, obtaining test results, completion of full documentation, and organ referral and allocation are completed after surgical retrieval. Geographic considerations and immediate staff availability are important in determining whether expedited donation is feasible, given the requirement for retrieval teams to travel in a short time frame to the donor hospital, with the risk of loss of donation potential entirely if the process cannot be expedited. At all phases of donation work-up, the DSNC continually re-evaluates whether a more standard pathway is possible either through donor stabilisation or changes in family requirements.

\section{Table 1. Key elements of the expedited pathway compared with the standard pathway}

\begin{tabular}{|c|c|c|}
\hline & Expedited pathway & Standard pathway \\
\hline Referral & $\begin{array}{l}\text { Donor physiological instability or family requirements for } \\
\text { expedited work-up identified }\end{array}$ & No indicators for expedited work-up on referral \\
\hline \multirow[t]{2}{*}{ Pre-hospital } & $\begin{array}{l}\text { DSNC discusses details with medical specialist on call;* } \\
\text { specifically seeks advice on physiological stability measures } \\
\text { (if relevant) }\end{array}$ & DSNC discusses details with Medical Specialist on call \\
\hline & Plan for two DSNCs to attend hospital & Usually one DSNC attends hospital \\
\hline \multirow[t]{2}{*}{ Hospital arrival } & $\begin{array}{l}\text { DSNC } 1 \text { meets with family on arrival to confirm consent and } \\
\text { explain the process }\end{array}$ & \multirow[t]{2}{*}{ DSNC meets with family to confirm consent } \\
\hline & $\begin{array}{l}\text { DSNC } 2 \text { organises essential blood tests (serology, tissue } \\
\text { typing, NAT tests) and organises retrieval }\end{array}$ & \\
\hline \multirow[t]{5}{*}{ Work-up } & $\begin{array}{l}\text { DSNC } 1 \text { holds a medical and social history interview } \\
\text { with family, in particular, determining if any potential } \\
\text { contraindications to donation exist. Work-up often continues } \\
\text { after retrieval }\end{array}$ & $\begin{array}{l}\text { Detailed medical and social history with family } \\
\text { together with collateral information from many } \\
\text { sources. DSNC documents the information } \\
\text { contemporaneously }\end{array}$ \\
\hline & \multirow{4}{*}{$\begin{array}{l}\text { DSNC } 2 \text { obtains legal consents (eg, from the Coroner), and } \\
\text { confirms retrieval start time with operating theatre at donor } \\
\text { hospital and with surgical retrieval team }\end{array}$} & $\begin{array}{l}\text { DSNC organises additional tests as requested by the } \\
\text { medical consultant and transplant units }\end{array}$ \\
\hline & & $\begin{array}{l}\text { DSNC obtains legal consents, organises logistics of } \\
\text { retrieval, chases test results (including serology, tissue } \\
\text { typing, NAT) }\end{array}$ \\
\hline & & $\begin{array}{l}\text { DSNC refers organs and receives acceptance before } \\
\text { retrieval }\end{array}$ \\
\hline & & Work-up usually complete before retrieval \\
\hline \multirow[t]{3}{*}{ Retrieval } & DSNC 1 collects data & Data collection, labelling and packaging \\
\hline & DSNC 2 manages labelling and packaging & \multirow{2}{*}{$\begin{array}{l}\text { Serology (with or without) NAT results always back } \\
\text { before retrieval }\end{array}$} \\
\hline & Serology and NAT results may not be back before retrieval & \\
\hline Post-retrieval & $\begin{array}{l}\text { Referral of organs, completion of medical and social history } \\
\text { interview as well as other documentation (as needed) }\end{array}$ & Post-retrieval follow-up \\
\hline
\end{tabular}

DSNC = donation specialist nursing coordinator; NAT = nucleic acid test. * The medical specialist on call is an intensive care specialist with donation expertise who provides advice regarding donor suitability, physiological management and other aspects of the donation process. 


\section{ORIGINAL ARTICLES}

\section{Methods}

We performed a retrospective audit of both the DonateLife electronic donor record and the DonateLife Victoria organ allocation database for all actual and intended donors in Victoria between 1 January 2015 and 31 December 2018. An "actual organ donor" was defined as a donor in whom the donation operation commenced (with surgical incision), irrespective of whether organs were utilised for transplantation - this is consistent with the Australia and New Zealand Organ Donation Registry (ANZOD) definition. ${ }^{7}$ On the other hand, "intended donors" were those for whom there was formal written consent for donation but donation did not occur. This may have either been because they did not die in a suitable time frame for donation after withdrawal of cardiorespiratory supports, a medical contraindication was identified during the work-up, cardiac arrest occurred before retrieval, or due to logistic impediments. In practice, donors were not always clearly identified a priori for expedited or standard pathway work-up. Therefore, for our study, we retrospectively grouped all actual organ donors into those for whom the time from formal written consent to commencement of the organ donation procedure (the work-up time) was less than or equal to 6 hours (expedited pathway donors), or greater than 6 hours (standard pathway donors). The analysis included a detailed review of case notes for donors who met the expedited pathway donor definition to further determine clinical characteristics and the reasons for the shortened process. We also further explored instances where circulatory death occurred within 6 hours of consent but donation did not eventuate (expedited pathway intended donors).

We explored kidney transplant recipient outcome data from actual donors, including the requirement for dialysis within 7 days after transplant, as well as graft and patient survival as reported to the Australia and New Zealand Dialysis and Transplant Registry (ANZDATA) to 31 December 2018. We liaised with the relevant transplant units for recipient outcomes for other solid organs donated. The study was reviewed by the Human Research Ethics Committee of the Australian Red Cross Blood Service (the organisation that hosts DonateLife Victoria) and was approached as a low/negligible risk project not requiring informed consent from donor's surrogate decision makers or recipients.

\section{Statistical analysis}

For continuous variables, data are presented as median and interquartile range (IQR). Differences between groups were assessed using the Mann-Whitney $U$ test for continuous data or the two proportion Z test for proportional data. The $\chi^{2}$ or Fisher exact tests (as appropriate) were used to compare categorical data. A two-sided $P<0.05$ was considered significant.

\section{Results}

\section{Demographic and clinical characteristics}

We identified a total of 38 expedited pathway and 569 standard pathway donors in the study period (Figure 1). Expedited donors, therefore, represented $6 \%$ of the total donor pool. All but three of the expedited donors were from metropolitan hospitals.

Table 2 lists the clinical and demographic characteristics of each group. The median work-up time (time from formal written consent to commencement of the retrieval procedure) was 3.7 (IQR, 2.7-4.5) hours for expedited pathway donors, compared with 23.4 (IQR, 18.7-37.7) hours for standard pathway donors. Of note, work-up times for standard pathway donors showed an increase year on year.

Expedited pathway donors proceeded to donation mostly via the donation after circulatory death (DCD) pathway (79\% $\checkmark 40 \%$ in the standard pool) and the profile of the cause of death was different, with a notably higher proportion of non-neurological conditions (eg, decompensated liver failure, lung graft rejection, from previous transplant; respiratory failure; and burns). There were proportionally more patients with blood group $A$ and fewer with blood group B in the expedited group. Comorbidities (hypertension, diabetes mellitus and smoking) were similar between groups. The numbers of vasoactive agents used as well as the proportions of donors on particular agents were

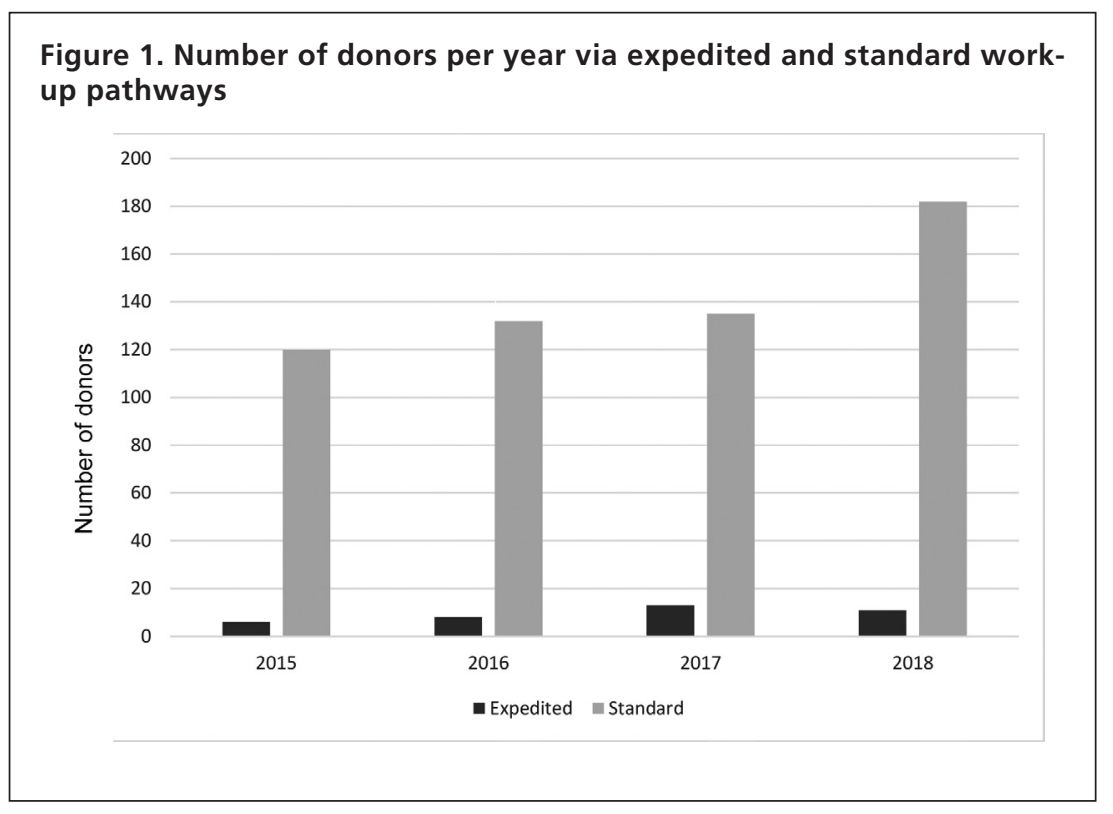


ORIGINAL ARTICLES

\begin{tabular}{|c|c|c|c|}
\hline & Expedited pathway & Standard pathway & $P$ \\
\hline Total number of donors & $38(6 \%)$ & $569(94 \%)$ & \\
\hline $\begin{array}{l}\text { Median work-up time (IQR), } \\
\text { hours }\end{array}$ & & & $<0.001$ \\
\hline $2015-2018$ & $3.7(2.7-4.5)$ & $23.4(18.7-37.7)$ & \\
\hline 2015 & $3.5(3.3-3.7)$ & $19.7(16.8-24.5)$ & \\
\hline 2016 & $3.5(2.4-4.5)$ & $21.9(18.4-29.3)$ & \\
\hline 2017 & $3.7(2.8-4.1)$ & $25.8(19.6-39.3)$ & \\
\hline 2018 & $4.5(2.2-4.8)$ & $26.5(20.5-40.9)$ & \\
\hline Median age (IQR), years & $51(31-61)$ & $50(36-61)$ & NS \\
\hline Median BMI (IQR) & $27(23-29)$ & $26(23-31)$ & NS \\
\hline Donation after circulatory death & $30(79 \%)$ & $228(40 \%)$ & $<0.001$ \\
\hline Sex, female & $19(50 \%)$ & $232(41 \%)$ & NS \\
\hline Cause of death & & & $<0.001$ \\
\hline Cerebral hypoxia/ischaemia & $15(39 \%)$ & $201(35 \%)$ & \\
\hline Intracranial haemorrhage & $8(21 \%)$ & $212(37 \%)$ & \\
\hline Ischaemic stroke & $1(3 \%)$ & $37(7 \%)$ & \\
\hline Non-neurological condition & $9(24 \%)$ & $20(4 \%)$ & \\
\hline Other neurological condition & $0(0 \%)$ & $13(2 \%)$ & \\
\hline Traumatic brain injury & $5(13 \%)$ & $86(15 \%)$ & \\
\hline Blood group & & & 0.038 \\
\hline $\mathrm{O}$ & $20(53 \%)$ & 277 (49\%) & \\
\hline A & $8(21 \%)$ & $218(38 \%)$ & \\
\hline$A B$ & $2(5 \%)$ & 17 (3\%) & \\
\hline B & $8(21 \%)$ & $57(10 \%)$ & \\
\hline
\end{tabular}

\begin{tabular}{|c|c|c|c|}
\hline \multicolumn{4}{|l|}{ Comorbidities } \\
\hline Hypertension & $9(24 \%)$ & $166(29 \%)$ & NS \\
\hline - Yes & $29(76 \%)$ & $399(70 \%)$ & NS \\
\hline - No & 0 & $4(1 \%)$ & NS \\
\hline - Unknown & 0 & $5(1 \%)$ & \\
\hline Diabetes mellitus & $4(11 \%)$ & $56(10 \%)$ & \\
\hline - Type 1 & $34(89 \%)$ & $508(89 \%)$ & \\
\hline - $\quad$ Type 2 & $11(29 \%)$ & $236(41 \%)$ & \\
\hline - No & $11(29 \%)$ & $123(22 \%)$ & \\
\hline Smoking status & $16(42 \%)$ & $207(36 \%)$ & \\
\hline - Current & 0 & $3(1 \%)$ & \\
\hline - Former & & $166(29 \%)$ & \\
\hline - Never & & 399 (70\%) & \\
\hline - Unknown & & $4(1 \%)$ & \\
\hline
\end{tabular}

small proportion of patients in each group received hormonal therapy (methylprednisolone or triiodothyronine).

Kidneys were the most common organ transplanted from expedited pathway donors, with small numbers of lung, liver and pancreas transplants. The mean number of organ transplants from expedited donors was 1.66 , less than half the value standard donors.

\section{Reasons for expedited donation}

Of the expedited pathway donors, most $(n=30)$ were due to reasons of physiological instability, such as circulatory failure, hypoxia or bleeding, while eight were due to needing to meet family requirements concerning the timing of the organ retrieval procedure.

Of the 30 physiologically unstable donors, 27 were receiving norepinephrine (median maximal dose, $0.62 \mu \mathrm{g} / \mathrm{kg} /$ min; IQR, 0.42-0.92), with 20 of these patients additionally receiving epinephrine and/or vasopressin. Of the remaining three donors in this group, one was receiving high dose metaraminol alone, another had refractory hypoxaemia without vasopressor requirements, and data were not recorded for the third donor. The only donor not receiving vasoactive agents was difficult to support due to refractory hypoxaemia. Six patients had extracorporeal or mechanical physiological supports, including two patients receiving continuous renal replacement therapy (CRRT), two

similar, except for a higher epinephrine use in the expedited group. Doses of agents and other support therapies (eg, extracorporeal life support) were only obtained for the expedited donor group and are described below. A similarly on extracorporeal life support (with CRRT), and two with an intra-aortic balloon pump in situ (one of these patients was also receiving (RRT). Two patients deteriorated to cardiac arrest before the planned time for retrieval and, in 


\section{ORIGINAL ARTICLES}

\section{Table 2. Clinical details of donors via the expedited and standard pathways (Continued)}

\begin{tabular}{|c|c|c|c|}
\hline & Expedited pathway & Standard pathway & $P$ \\
\hline Number of vasoactive agents & & & NS \\
\hline On any agent & $36(95 \%)$ & $509(89 \%)$ & \\
\hline One agent & $9(24 \%)$ & $207(36 \%)$ & \\
\hline Two agents & $17(45 \%)$ & $194(34 \%)$ & \\
\hline Three agents & $9(24 \%)$ & $89(16 \%)$ & \\
\hline Four agents & $1(2 \%)$ & $15(3 \%)$ & \\
\hline Five agents & 0 & $4(1 \%)$ & \\
\hline $\begin{array}{l}\text { Not receiving vasoactive } \\
\text { agent }\end{array}$ & $2(5 \%)$ & $60(11 \%)$ & \\
\hline \multicolumn{4}{|l|}{ Type of vasoactive agent } \\
\hline Norepinephrine & $32(84 \%)$ & $484(85 \%)$ & NS \\
\hline Vasopressin & $19(50 \%)$ & $226(40 \%)$ & NS \\
\hline Epinephrine & $18(47 \%)$ & $165(29 \%)$ & 0.04 \\
\hline Dobutamine & $2(5 \%)$ & $8(1 \%)$ & NS \\
\hline Dopamine & 0 & $1(<1 \%)$ & NS \\
\hline \multicolumn{4}{|l|}{ Receiving hormonal agents } \\
\hline Methylprednisolone & $2(5 \%)$ & $15(3 \%)$ & NS \\
\hline Tri-iodothyronine & $1(3 \%)$ & $43(8 \%)$ & NS \\
\hline \multicolumn{4}{|l|}{$\begin{array}{l}\text { Organs transplanted from } \\
\text { donors, }{ }^{*} n\end{array}$} \\
\hline Kidney & 55 & $942^{+}$ & \\
\hline Liver & 3 & $264^{\ddagger}$ & \\
\hline Lung & 4 & $523^{\S}$ & \\
\hline Pancreas & 1 & 65 & \\
\hline Heart & 0 & 116 & \\
\hline Intestine & 0 & 3 & \\
\hline Organs per donor, mean & 1.66 & 3.36 & \\
\hline
\end{tabular}

$\mathrm{BMI}=$ body mass index; $\mathrm{DCD}=$ donation after circulatory death; IQR = interquartile range; NS = not significant. * Kidneys counted as two separate transplants from one donor. Lungs counted as two separate transplants from one donor. † Includes eight en-bloc or double adult kidney transplants. ₹ Includes 243 whole liver and 21 split liver transplants. § Includes 248 double lung and 31 single lung transplants.

supported and died within 3 hours of consent. Three expedited pathway intended donors died beyond 90 minutes from withdrawal of cardiorespiratory supports such that DCD was abandoned. Two of these patients had expedited work-up due to family requests, while the other patient, who had expedited work-up due to concerns about ability to sustain support on non-invasive ventilation did not die within 90 minutes of removal of non-invasive ventilation.

\section{Expedited pathway donors and organ utilisation}

Expedited donors donated 78 organs for transplantation (70 kidneys, four lungs, three livers, one pancreas). Of the 38 expedited donors, 35 were kidney donors, with two being multivisceral donors, one being a lung-only donor and one a liver-only donor. One patient was found to have extensive intraabdominal ischaemia at operation and the planned kidney retrieval did not occur (included in the "actual donor" pool consistent with our definition above).

In total, 70 kidneys were retrieved, with 15 kidneys not utilised for transplant (non-utilisation rate of $21 \%$ ). The reasons for nonutilisation included non-acceptance of all offers on the National Organ Matching System (NOMS) (eight grafts), ischaemic damage with organs deemed unsuitable for

both cases, surgical retrieval teams were rapidly assembled to enable donation to proceed, with one patient receiving cardiopulmonary resuscitation.

There were eight occasions where donation was expedited due to family request, mostly due to a DSNC and/ or treating team assessment that family were not willing to prolong the intensive care period for the usual length of time required for donation work-up. Other reasons included the desire to avoid the occurrence of death on a family member's birthday and a religious requirement.

There were four expedited pathway intended donors during the period. One patient could not be physiologically transplant (four), and medical contraindications determined post retrieval (three).

Of the patients receiving extracorporeal or other mechanical physiological supports, only three kidneys (out of 12) were not utilised. These included two kidneys from a donor receiving extracorporeal life support and CRRT where the reason for non-utilisation was a medical contraindication discovered that was unrelated to perfusion of the kidneys. The kidney non-utilisation rate was significantly higher than for the non-expedited kidney donor pool (21\% v 3.7\%; $P<0.001$ ). The lungs, livers and pancreas retrieved were all transplanted. In addition to solid organs, tissue was also 


\section{ORIGINAL ARTICLES}

\section{Table 3. Comorbidities and renal indices for expedited and standard pathway donated kidneys}

\begin{tabular}{lccc} 
& $\begin{array}{c}\text { Expedited } \\
\text { pathway kidneys }\end{array}$ & $\begin{array}{c}\text { Standard pathway } \\
\text { kidneys }\end{array}$ & $\boldsymbol{P}$ \\
\hline $\begin{array}{l}\text { Total number of } \\
\text { transplanted organs }\end{array}$ & 55 & 934 & \\
$\begin{array}{l}\text { Median admission } \\
\text { creatinine (IQR), } \mu \mathrm{mol} / \mathrm{L}\end{array}$ & $86(66-128)$ & $78(64-104)$ & 0.049 \\
$\begin{array}{l}\text { Median terminal creatinine } \\
\text { (IQR), } \mu \mathrm{mol} / \mathrm{L}\end{array}$ & $99(72-204)$ & $71(58-103)$ & $<0.001$ \\
\begin{tabular}{l} 
Median KDPI (IQR), \% \\
\hline
\end{tabular} & $57 \%(32-76 \%)$ & $49 \%(26-75 \%)$ & $\mathrm{NS}$ \\
\hline
\end{tabular}

$\mathrm{IQR}=$ interquartile range; $\mathrm{KDPI}=$ Kidney Donor Profile Index; NS = not significant.

surgery for kidney (and occasionally other solid organs) donation within 6 hours of consent when required by physiological instability or family needs. Moreover, we found that in most of these patients, successful organ retrieval and subsequent transplantation occurred. Finally, graft and patient survival in recipients from these donors were satisfactory.

\section{Relationship to previous studies}

For the broader donor pool in our study, the time from consent to retrieval (around 24 hours) is comparable to data from the United Kingdom ${ }^{9}$ and Canada. ${ }^{10}$ Recommendations on donor physiological

commonly retrieved from this group: 19 donors donated eye tissue, seven donated cardiovascular tissue, five donated musculoskeletal tissue and four donated skin.

\section{Transplant recipient outcomes}

Donor renal indices were recorded for the 55 kidney donations that resulted in a transplant (Table 3). The admission and final measured creatinine were higher in the expedited group. The Australian Kidney Donor Profile Index $(\mathrm{KDPI})^{8}$ (a percentile ranked from $1 \%$ to $100 \%$ ), a measure of kidney quality using donor parameters such as age, cause of death, creatinine, history of hypertension or diabetes, height and weight, and donor pathway (donation after brain death or DCD), was not significantly different between groups.

Short term recipient and graft survival was available until the end of 2018 (to 48 months; median, 490 days (IQR, 320-1006). Recipients from expedited pathway donors experienced delayed graft function (requirement for dialysis within 7 days of transplant) in a significantly higher proportion $(71 \% \vee 38 \% ; P<0.001)$, but this did not translate into inferior short or medium term graft or recipient survival (Figure 2). Only three kidney recipients from the expedited pool experienced graft failure (all within the first year after transplantation) and two of these patients subsequently died. All three liver recipients were alive at the end of 2018 without need for retransplantation. Of the two lung recipients, one died at day 622 of chronic rejection.

\section{Discussion}

\section{Key findings}

In our retrospective study of Victorian donors from 2015 to 2018 , we found that it was feasible to proceed to management during this phase have been the subject of consensus statements and guidelines in Australia and elsewhere. 5,11 Maintaining homeostasis by meeting physiological goals is associated with a greater number of organs donated in observational studies of standard ${ }^{12}$ and expanded criteria ${ }^{13}$ donors, while a randomised controlled trial of protocolised fluid therapy in brain dead donors did not demonstrate overall benefit. ${ }^{14}$ In most donors in these studies, physiological goal bundles could not be met in entirety at any time in the process, including immediately before organ procurement. In our study, clinicians either deemed it impossible to maintain physiological stability for long enough to complete standard work-up, or family circumstances or requirements did not permit this time. In these extreme situations, we have shown that with a focus on proceeding to retrieval as quickly as possible, such patients can still be considered for organ and tissue donation.

A byproduct of our study is the observation that the standard work-up period for donors has increased over time in Victoria. Such increases have also been noted in the United Kingdom. ${ }^{9}$ Potential factors could include increasing clinical complexity in an expanded donor pool requiring longer work-up or altered logistic factors. Family discomfort with the length of the organ donation process has been reported as a reason for family refusal in Victoria ${ }^{15}$ and elsewhere. ${ }^{16,17}$ It is possible that the lengthening of the organ donation process over time may lead to more families declining donation. Expediting the process permits donation in such circumstances, even if largely restricted to the kidneys.

Most expedited pathway donors were kidney-only donors. With kidney donation, prolonged cold ischaemic time, while not desirable, does not necessarily preclude transplantation. ${ }^{18}$ Therefore, donation-related processes such as medical history gathering, test processing and organ 


\section{ORIGINAL ARTICLES}

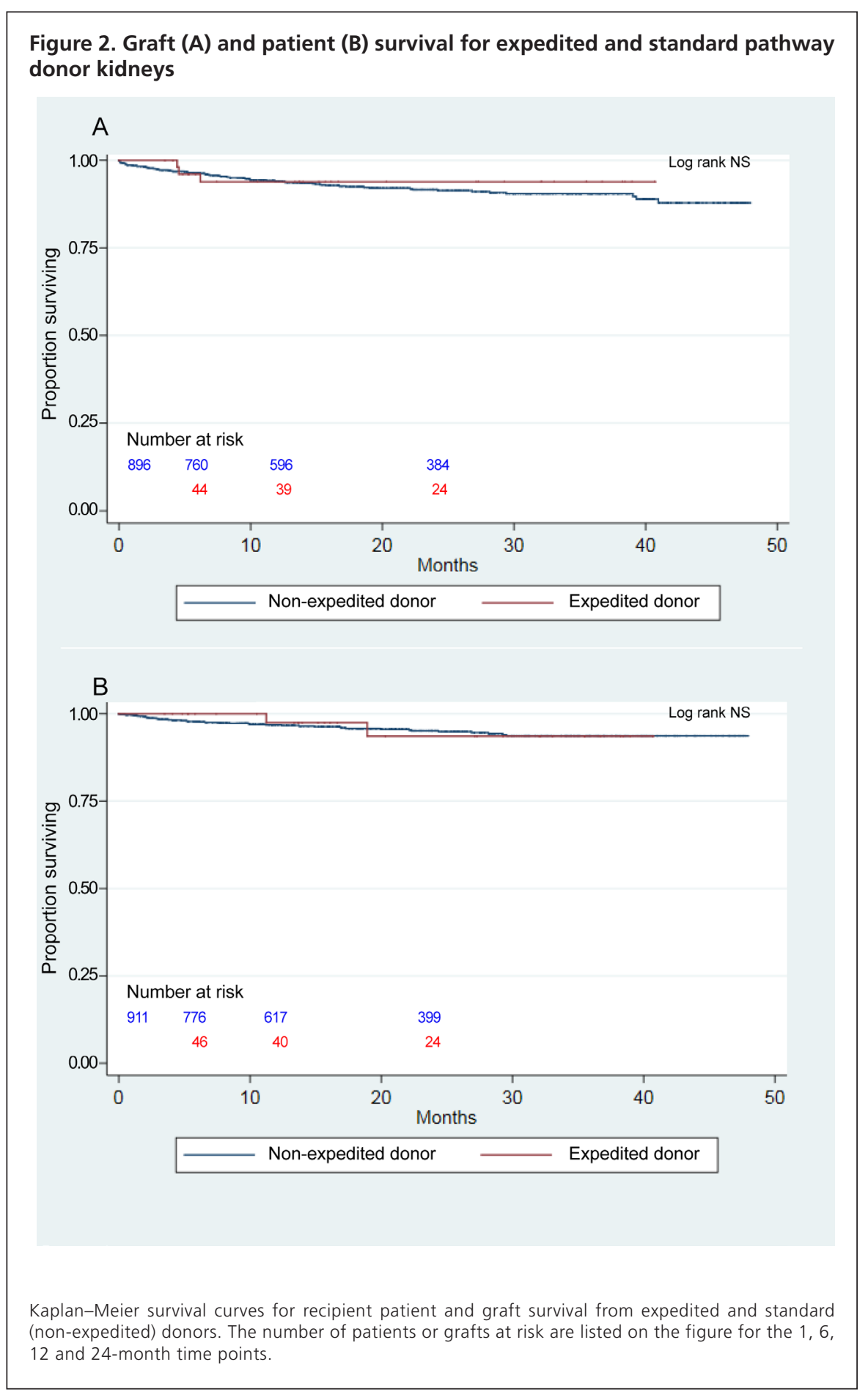

referral can continue for many hours after retrieval. For heart, lung and liver donation, shorter periods of cold ischaemia are generally tolerated, ${ }^{3}$ severely limiting the time available for these tasks. Nonetheless, liver and lung donation was still possible in a small number of these donors.

As described, the kidney non-utilisation rate was higher for expedited pathway donors. A recent study examined donor clinical and other factors that account for the rising, but still small, non-utilisation rate in Australia (5.8\% for the period 20132017). ${ }^{19}$ Of note, the rate of organ non-utilisation in expedited donors in our study is comparable with overall rates reported in the United States in recent years. ${ }^{20}$

\section{Study implications}

Our findings imply that, in patients for whom continued vital organ support for the typical 24-hour period between consent and donation is considered impossible, an expedited donation protocol can deliver successful organ procurement in most cases. In our donor population, the majority of opportunities for expedited donation occurred in the form of DCD. Importantly, kidneys donated and transplanted under such circumstances ultimately recover to a degree sufficient to enable dialysis independence.

\section{Study strengths and limitations}

To our knowledge, this is the first study to specifically describe how donation remains possible in patients for whom the treating clinicians believe that vital organ support for the typical 24-hour period necessary to perform standard organ donation preparation is not possible. We have also described the use of an expedited approach in a small cohort of donors for family requirements and where the alternative may have been refusal of consent. In both situations, we have shown that organ (especially kidney) retrieval was feasible with satisfactory transplantation outcomes. These findings have implications for potential expansion of the donor pool in circumstances where donation may otherwise have been ruled out.

We acknowledge some limitations. Firstly, we have made the assumption that for the expedited cases a standard approach would have resulted in total loss of donation opportunity. It is not possible to determine if more aggressive efforts at stabilisation would have been successful and therefore more non-renal organs could have been donated. We think, however, this is unlikely in the context of current Australian practice, with intensivist-led 


\section{ORIGINAL ARTICLES}

management and low rates of failed physiological support. ${ }^{6}$ Similarly, for the group of patients for whom family requirements triggered expedited donation, it is possible that the family may have been persuaded to wait longer. However, DSNCs are specifically trained in exploring the family's comfort with slowing down the process if feasible and assessing the risk of the family withdrawing their consent in these situations. Thus, it seems more likely that the alternative to expedited donation in these cases would have been withdrawal of consent.

Given we retrospectively identified donors whose workup was expedited, we did not obtain data for those patients who may have initially been considered in need of the expedited pathway and then either stabilised physiologically or had changed circumstances which permitted a longer work-up. Donors in this category would potentially be resource-intensive for the system, but may not have been referred in the absence of an available expedited pathway.

We did not evaluate kidney recipient clinical or demographic characteristics so it is possible that graft and recipient survival was influenced by differences between recipient groups. Our aim was to demonstrate meaningful donation outcomes from the small expedited cohort rather than to systematically compare these with the broader group, and we therefore used unadjusted outcomes. Even though non-utilisation of retrieved kidneys was higher, the non-utilisation rate was comparable to that reported in the United States. ${ }^{20}$ Finally, we acknowledge that the expedited donation group was a highly selected cohort with specific clinical and logistic requirements in each case and it is neither prudent nor possible to expedite all donation cases.

\section{Conclusion}

Expedited pathway donation is feasible with acceptable donation outcomes if the expected alternative is the donation not proceeding. Clinicians should consider the possibility of expedited donation when physiological instability or family requirements preclude standard organ donation work-up times.

Acknowledgements: We thank the Australia and New Zealand Dialysis and Transplant Registry (ANZDATA) for providing transplant outcome data. The interpretation and reporting of these data are the responsibility of the authors and in no way should be seen as an official policy or interpretation of ANZDATA. We thank Christopher Davies from ANZDATA for additional assistance with the production of graft and recipient survival curves. We would also like to acknowledge the work of the Donation Specialist Nursing Coordinators who are not only pivotal to the donation process but are also responsible for recording donor data which was utilised in this study.

\section{Competing interests}

Rohit D'Costa, Samuel Radford and Leanne McEvoy are employees of DonateLife Victoria (part of Australian Red Cross LifeBlood), which coordinates organ and tissue donation in the State of Victoria, Australia. Helen Opdam is an employee of the Organ and Tissue Authority, which is an independent statutory agency within the Australian Government Health portfolio

\section{Author contribution}

All authors contributed to the concept and outline of the article. RLD and MM extracted and analysed the data. RLD wrote the draft and all authors contributed to the revision and finalisation of the manuscript.

\section{Funding}

No external funding was received.

\section{Author details}

Rohit L D'Costa ${ }^{1,2}$
Samuel Radford $1,3,4$
Helen I Opdam 3,5
Mark McDonald5
Leanne McEvoy ${ }^{13}$
Rinaldo Bellomo $2,3,4$
1 DonateLife Victoria, Melbourne, VIC, Australia.
2 Melbourne Health, Melbourne, VIC, Australia.
3 Austin Health, Melbourne, VIC, Australia.
4 University of Melbourne, Melbourne, VIC, Australia.
5 Organ and Tissue Authority, Canberra, ACT, Australia.

Correspondence: rohit.dcosta@mh.org.au

\section{References}

1 Australia and New Zealand Organ Donation Registry. 2019 Annual report, section 2: overview of organ donation activity in Australia and New Zealand. Adelaide: Australia and New Zealand Dialysis and Transplant Registry, 2019. https://www. anzdata.org.au/report/anzod-annual-report-2019/ (viewed Sept 2020).

2 Australia and New Zealand Dialysis and Transplant Registry. 41st Report, chapter 6: Australian transplant waiting list. Adelaide: ANZDATA, 2019. https://www.anzdata.org.au/wp-content/ uploads/2018/11/c06_waiting-list_2017_v1.0_20190426-2. pdf (viewed Sept 2020).

3 Organ and Tissue Authority. Best practice guideline for offering organ and tissue donation in Australia. https://donatelife.gov. au/resources/clinical-guidelines-and-protocols/best-practiceguideline-offering-organ-and-tissue (viewed Sept 2020). 


\section{ORIGINAL ARTICLES}

4 Transplantation Society of Australia and New Zealand. Clinical guidelines for organ transplantation from deceased donors, $v$ 1.4,2020, 2019. https://www.tsanz.com.au/TSANZ_Clinical_ Guidelines_Version\%201.4.pdf (viewed Sept 2020).

5 Australian and New Zealand Intensive Care Society. The statement on death and organ donation; edition 4, 2019. https://www.anzics.com.au/wp-content/uploads/2020/07/ ANZICS-Statement-on-Death-and-Organ-Donation-Edition-4. pdf (viewed Sept 2020).

6 Australia and New Zealand Organ Donation Registry. 2019 Annual report, section 3: deceased organ donor pathway. Adelaide: Australia and New Zealand Dialysis and Transplant Registry, 2019. https://www.anzdata.org.au/report/anzodannual-report-2019/ (viewed Sept 2020).

7 Australia and New Zealand Organ Donation Registry. Monthly report on deceased organ donation in Australia, September 2019. https://www.anzdata. org.au/wp-content/uploads/2019/10/20191004_

ANZODMonthlyReport_2019September.pdf (viewed Sept 2020).

8 Transplantation Society of Australia and New Zealand. A guide to the Australian Kidney Donor Profile Index (KDPI). TSANZ, 2016. https://www.tsanz.com.au/standalonepages/documents/ AustralianKDPIINFOv1.0.pdf (viewed Sept 2020).

9 National Organ Donation Committee. Minutes of the National Organ Donation Committee; 5 June 2018. https://www. odt.nhs.uk/odt-structures-and-standards/clinical-leadership/ national-organ-donation-committee (viewed Sept 2020).

10 D'Aragon F, Cook D, Dhanani S, et al. Hamilton-DONATE: a city-wide pilot observational study of the ICU management of deceased organ donors. Can J Anaesth 2018; 65: 1110-9.

11 Kotloff RM, Blosser S, Fulda GJ, et al. Management of the potential organ donor in the ICU: Society of Critical Care
Medicine/American College of Chest Physicians/Association of Organ Procurement Organizations Consensus Statement. Crit Care Med 2015; 43: 1291-325.

12 Malinoski DJ, Patel MS, Daly MC, et al. The impact of meeting donor management goals on the number of organs transplanted per donor: results from the United Network for Organ Sharing Region 5 prospective donor management goals study. Crit Care Med 2012; 40: 2773-80.

13 Patel MS, Zatarain J, De La Cruz S, et al. The impact of meeting donor management goals on the number of organs transplanted per expanded criteria donor: a prospective study from the UNOS Region 5 Donor Management Goals Workgroup. JAMA Surg 2014; 149: 969-75.

14 Al-Khafaji A, Elder M, Lebovitz DJ, et al. Protocolized fluid therapy in brain-dead donors: the multicenter randomized MOnIToR trial. Intensive Care Med 2015; 41: 418-26.

15 Neate SL, Marck CH, Skinner M, et al. Understanding Australian families' organ donation decisions. Anaesth Intensive Care 2015; 43: 42-50.

16 Siminoff LA, Lawrence RH. Knowing patients' preferences about organ donation: does it make a difference? J Trauma 2002; 53: 754-60.

17 Vincent A, Logan L. Consent for organ donation. Br J Anaesth 2012; 108 (Suppl): i80-7.

18 van der Vliet JA, Warle MC. The need to reduce cold ischemia time in kidney transplantation. Curr Opin Organ Transplant 2013; 18: 174-8.

19 Sypek MP, Ullah S, Hughes PD, et al. Examining the increased rates of deceased donor kidney nonutilization in Australia: what has changed? Transplantation 2019; 103: 2582-90.

20 Mohan S, Chiles MC, Patzer RE, et al. Factors leading to the discard of deceased donor kidneys in the United States. Kidney Int 2018; 94: 187-98. 